\title{
Emotionale Rationalität und Planung: Planungsansätze einer ,3. Generation“ \\ Oder: Zum depolitisierenden Potenzial von Vertrauensbildung und Selbstversicherung
}

\author{
Axel Schubert
}

Dem Beitrag liegt die Annahme zugrunde, dass der Umgang mit Verunsicherungen, die den planerischen Fortschritt zu unterbrechen drohen, im Planungshandeln einen zentralen Stellenwert einnimmt. Darum ist es planungspraktisch so wichtig, bei allen Beteiligten Vertrauen für die Annahmen und ,Produkte' einer Planung (Pläne, Leitbilder etc.) zu gewinnen. Emotionstheoretisch werden Verunsicherungen und Irritationen dagegen als Bedingung von Rationalität, Reflexivität und von Lernprozessen angesehen. Vertrauensbildende Effekte von Planungen sind daher immer dann als kritisch anzusehen, wenn durch sie Reflexion ausbleibt und damit ,das Politische' aus dem Blickfeld zu geraten droht. Mit meinem Beitrag leuchte ich aus, wie Planungshandeln im Umgang mit Verunsicherung verstanden werden kann. Dazu hinterfrage ich bestehende Konzeptionen und fokussiere auf die Rolle von Emotionen im (Planungs-)Handeln. Mit Bezug auf eine ,emotionale Rationalität' schlage ich Planungsansätze einer ,dritten Generation' vor und skizziere deren Bedeutung für eine kritische Planungspraxis. Zugleich möchte ich zu einem emotional turn in der Planungstheorie beitragen.

Ersteinreichung: 1. Juni 2013; Veröffentlichung online: 5. Mai 2014

An english abstract can be found at the end of the article.

\section{Einleitung}

In der Diskussion um die ,postpolitische Stadt' wird auch in der Raumplanung Handlungsbedarf ausgemacht. So fordert beispielsweise Peter Marcuse (2013: 9) einen kritischen neuen Blick auf Stadt im Sinne ihrer Reorganisation und eines re-imagining. An die Vorstellung einer Reimagination möchte ich anschließen - nicht jedoch, um hier ein alternatives Bild von Raumproduktion zu entwerfen, sondern bezüglich der theoretischen Konzeption von emotionalen Aspekten planerischen Handelns. Mit so einem neuen Blick entwickele ich bisherige Planungsansätze zu solchen einer ,dritten Generation' weiter. Dabei grenze ich mich von der von Walter Schönwandt vorgeschlagenen, dritten Generation“ klar ab.

Eine Wende hin zu einer konzeptionellen Berücksichtigung von emotionalen Dimensionen im Handeln und Verstehen wird in den Sozial,Geistes- und Naturwissenschaften seit einigen Jahrzehnten diskutiert, dennoch besteht bis heute Skepsis, ob bereits von einem emotional turn gesprochen werden kann (Schnabel 2012: 9). Dabei spielen Emotionen weder in der Politikwissenschaft noch in der Politischen Psychologie 
eine bedeutende Rolle (Nullmeier 2006: 84 f.) und stehen als soziale und analytische Kategorien erst am Beginn ihrer Erforschung (HammerTugendhat/Lutter 2010: 10). Umgekehrt interessiert sich die Forschung $z u$ Emotionen noch wenig dafür, wie Handeln jenseits der etablierten Konzepte strategischen und/oder normbefolgenden Handelns aussehen könnte (Pettenkofer 2012: 201). Weder gelten die Zusammenhänge zwischen Emotionen und Handeln als ausreichend erklärt, noch liegt eine allgemein geteilte Theorie vor, mit der Emotionsursache, -entstehung oder -definition einheitlich beschrieben würden (Rothermund/Eder 2011: $196 \mathrm{f}$., Schützeichel 2012a: 231 ff., 2012b: 475). Ich zeige auf, weshalb Emotionen im Planungshandeln dennoch eine nicht zu vernachlässigende Kategorie darstellen, und weise auf eine emotionstheoretische Lücke bisheriger Planungstheorien hin. Zugleich möchte ich dazu beitragen, sie aufzufüllen.

Im ersten Kapitel greife ich auf die planungstheoretischen Arbeiten von Rittel (1992)[1] und Reuter (2000) zurück, um eine für Planung anzunehmende politische Rationalität auszuweisen. Für ein besseres Verständnis von Emotionen verweise ich in Kapitel 2 auf die unauflösbare Bedingtheit von Rationalität und Emotionalität. Im dritten Kapitel hinterfrage ich kritisch, wie in bestehenden planungstheoretischen Beiträgen Unsicherheiten und Widersprüchlichkeiten konzeptualisiert werden. Schließlich stelle ich Planungsansätze und -zugänge einer ,dritten Generation“ vor, die das Emotionale integrieren. In einem Ausblick ziehe ich mögliche Konsequenzen für eine kritische Planungspraxis.

Mit Planung beziehe ich mich primär auf (lokal-)staatliche Raumplanung. Der empirische Gehalt des Gesagten gründet in eigenen Erfahrungen in der Planungs- und Bewegungspraxis, die ich mit bestehender Theoriebildung induktiv abgleiche. Der Anspruch des Beitrags besteht darin, Erkenntnisse und Interpretationen aus der Praxis einem wissenschaftlichen, politischen und planungspraktischen Diskurs anzubieten. Selbstredend gibt es ,die Praxis nicht. Speziell in Bezug auf Emotionen stellt sie sich allen Planenden einzigartig dar. Genauso einzigartig sind damit auch die diesbezüglichen Ausgangspunkte planerisch-politischen Handelns.

\section{Rationalitätsverständnisse bisheriger Planungsansätze}

Aus planungslogischer Sicht ist ein Beitrag zur Lösung eines Problems wenn er mehr sein will als ein Zufallstreffer - erst dann möglich, wenn ausreichende Klarheit über das zu lösende Problem besteht. Konstitutiv für ein Problem ist dabei eine Diskrepanz zwischen einem gegebenen Ist und einem besseren Soll. Es mit Mitteln der Planung zu lösen, meint eine hinreichend gute Strategie zu entwickeln, mit deren Umsetzung beansprucht wird, die Diskrepanz zu schließen.

Der Planungstheoretiker Horst W.J. Rittel (vgl. Rittel 1977, Rittel/ Kunz 1972) hat in den $1970 e r$ Jahren herausgearbeitet, dass bei Planungsentscheidungen notwendigerweise auf unterschiedliches Wissen zurückzugreifen ist: auf faktisches (was ist?), deontisches (was soll sein?), instrumentelles (wie kann verändert werden?), explanatorisches (wie können Folgen erklärt werden?) und konzeptionelles Wissen (was meine ich, wenn ich Begriffe und Konzepte verwende?). Rittel zeigt auf, dass jedoch für „alle wesentlichen 
Planungsprobleme“ das Wissen, das planungslogisch zur ausreichenden Behandlung eines Problems erforderlich wäre, weder planungspraktisch noch erkenntnistheoretisch abschließend zusammengetragen werden kann (Rittel 1972: 43). Da Fragen in Bezug auf diese Wissensformen nicht eindeutig beantwortet werden können, bezeichnet Rittel Planungsprobleme als „bösartig“ (ebd.). Eine der zentralen Eigenschaften dieser Bösartigkeit liegt in der unauflösbaren Abhängigkeit von Problemformulierung und Problemlösung: Ohne abschließendes Wissen, wie die Diskrepanz zwischen Ist und Soll genau überwunden werden sollte, kann nämlich nicht behauptet werden, bereits ein ausreichendes Verständnis des Problems selbst und seiner tatsächlichen Ursachen gefunden zu haben (Rittel/ Webber 1973: 21 ff.). Planungsprobleme können darum nicht technisch respektive, richtig“ im Sinne einer technisch-wissenschaftlichen Rationalität gelöst werden - wie dies bei „zahmen“ (ebd.) Problemen der Fall ist -, sondern nur durch argumentative Prozesse, und das bedeutet: sozial. Die Übereinkunft über das, was (vorläufig) als Problem verstanden werden soll, bedarf also eines (gesellschaftlichen) Diskurses. So wird Planung von Rittel als im Kern politisch verstanden. Dabei fordert er, dass durch „Objektifizierung“ sowohl die zugrunde liegenden Zielvorstellungen als auch die Grundlagen für die jeweiligen Urteile der Beteiligten transparent zu machen sind (Rittel 1972: 49 ff.). Rittel versteht den/die Planer_in als „Hebamme für Probleme“ und lehnt die Proklamation von Sachzwängen, da sie „verdummen“, entschieden ab (ebd.: 52, Rittel 1976: 281). In der Überwindung der technischen Rationalität als planerisches Fundament sieht er den Übergang von Planungsansätzen der ersten zu auf Argumentation gründenden Ansätzen der zweiten Generation (Rittel 1972: 48 ff.).

Während Rittel damit wesentliche Grundlagen für ein Verständnis von Planung im Sinne einer diskursiven Rationalität und letztlich auch für einen communicative turn in der Planung formulierte, hat er zu Fragen der Macht im Rahmen der von ihm empfohlenen argumentativ-deliberativen Prozesse keine eigenen Beiträge geliefert (Fischbach et al. 1992: 411). Beide Aspekte bringt der Herausgeber von Rittels Schriften, Wolf D. Reuter, mit der von ihm beschriebenen „Komplementarität von Diskurs und Macht in der Planung“" (Reuter 2000) zusammen. Er zeigt auf, dass zwischen beiden eine „untrennbare Verkopplung“ besteht: Akte der Macht und diskursive Akte sind als permanent und unauflösbar aufeinander bezogen zu verstehen, unter anderem weil Akte der Macht durch Sprechakte aufgedeckt, diskursive Akte hingegen durch Akte der Macht eingeschränkt werden können (ebd.: 13 f.). Reuter folgert, dass die Rationalität von Planung in der Komplementarität der Konzepte von Macht und Diskurs zu suchen ist und damit jener der Politik gleicht. Damit verweist er auf „die aktive politische Rolle des Planers“ (ebd.), die auch darin besteht, sich in Auseinandersetzungen Macht bewusst zu bedienen; es gibt keine machtfreien Diskurse oder Diskurse, in denen Macht gleich verteilt ist, sondern nur machtverzerrte Diskurse.[2] Bei Planung ist daher von einem Konzept einer politischen Rationalität auszugehen.

Mit den beiden Rationalitäten - technisch-wissenschaftlicher und politischer - ist nun ein doppeltes Spannungsverhältnis gegeben. Einerseits kann Planung als ein Beitrag zu einem Schließprozess zwischen einem Ist 
und Soll konzeptualisiert werden, wobei aufgrund der Bösartigkeit von Planungsproblemen Ist, Soll und die entwickelte Strategie des Schließens nicht abschließend exakt, sondern nur vage im Rahmen einer gesellschaftlichen Übereinkunft definierbar sind. Solange wie das Aushandeln solcher Übereinkünfte für Akteure Unsicherheiten mit sich bringt und von ihnen als bedrohlich wahrgenommen wird, ist auch die Stabilität der Übereinkünfte selbst bedroht, da sie - die entsprechende Artikulationsfähigkeit und -stärke von Betroffenen vorausgesetzt - von diesen (erneut) infrage gestellt werden können. Im Umgang mit Unsicherheiten spielt Vertrauen eine bedeutende Rolle. Vertrauen muss daher als ein wesentliches Kriterium für erfolgreiche Planung verstanden werden. Es ermöglicht, dass zumindest vorläufige planerische Setzungen getroffen werden können, obwohl die im Rahmen des Planungsprozesses ausgehandelten Gewissheiten nur relativ sein können und damit immer verbleibende Ungewissheiten beinhalten. Gerade weil durch solche Setzungen Alternativen ausgeschlossen werden, weil sozioökologischen Interventionen ein totalitäres, das Demokratische außer Kraft setzendes Moment innewohnt (Swyngedouw 2009: 386) und weil durch demokratische Entscheide Ausschlüsse produziert werden (Marchart 2010: 362 ff.), kann Vertrauen in die planerischen Übereinkünfte als Ressource verstanden werden, durch die sich die jeweiligen Ausschlüsse und die Art, wie mit ihnen umgegangen wird, als akzeptabel präsentieren.[3]

Um die andere Seite des Spannungsverhältnisses zwischen den Rationalitäten in den Blick zu bekommen, beziehe ich mich nochmals auf Rittel. Rittel (1977: 119 ff., 1972: 54 ff.) weist nicht nur die bereits erwähnten fünf Urteilsformen aus, er stellt zudem „spontane“ oder „intuitive“ Urteile „deliberierten“ oder „überlegten“ gegenüber. Zudem verweist er auf den erkenntnistheoretisch begründeten und im Sinne einer Verstandesratio grundlegend irrationalen Charakter von Planung, da alles Urteilen letztendlich auf Spontanurteilen gründet:

„Dabei werden Spontanurteile auf der jeweils nächsten Deliberierungsstufe nicht notwendig einfacher, sondern eher immer grundsätzlicher und damit schwieriger. Je besser man ein Urteil begründen will, umso mehr muss man sich auf Unbegründetes, Ungerechtfertigtes, NichtAbgeleitetes verlassen“ (Rittel 1977: 119), denn „die Endpunkte sind immer Spontanurteile“ (Rittel 1972: 57).

Die Bösartigkeit hat also zur Folge, dass es nicht nur gesellschaftlich nicht möglich ist, zu eindeutigen respektive richtigen Lösungen $\mathrm{zu}$ gelangen, sondern dass dies auch Planenden nicht mit dem Anspruch auf Verstandesrationalität gelingen kann. Damit können sich Planende grundsätzlich nicht sicher sein, ob die von ihnen ins Spiel gebrachten Strategien angemessene Antworten in gegebenen Situationen sind oder nur die für sie im jeweiligen Moment naheliegenden. Was gegenüber Dritten Vertrauensbildung darstellt, ist für Planende damit die Selbstversicherung, die subjektiv-inneren Deliberationsprozesse an einem Punkt beendet zu haben, der in der jeweiligen Situation ausreichende Stimmigkeit vermitteln konnte.[4] Reuter fokussiert nun zuvorderst auf Macht, mit der einem fortschreitenden Deliberationsprozess in Planungsdiskursen etwas entgegengesetzt wird - indem zum Beispiel Diskurse eingeschränkt oder 
abgebrochen werden. Damit legt er ein Verständnis von Planenden nahe, die im Vollbesitz jener Kompetenzen sind, durch die ihnen mit normativstrategischem Kalkül ein zweckrationales Handeln auch tatsächlich gelingt. Wie sinnvoll ist aber die Annahme, dass Planende in erster Linie strategischintentional handeln? Wie gehen Planende mit den sich ihnen selbst aus der Bösartigkeit ergebenden Unsicherheiten um? Wie wirken sich Momente der Verunsicherung und Selbstversicherung auf das Agieren von Planenden aus, die ihr Tun in einem politischen Sinne verantwortlich wahrnehmen möchten? Wie gehen sie damit um, Verhandlungspartner, politische Auftragnehmer, Vorgesetzte/ Mitarbeitende, Angestellte, Planende mit eigenen Idealen und Politikberatende zugleich zu sein und zudem im eigenen, privaten Lebensvollzug zu stecken? Kurzum: Handeln Planende auch nach intentionalen Motiven, die nicht strategisch am Zweck der Planung orientiert sind - und wie könnten diese konzeptualisiert werden?

\section{Handeln im Lichte einer, emotionalen Rationalität}

Ein Schlüssel für die Beantwortung der aufgeworfenen Fragen liegt meines Erachtens in der Berücksichtigung von emotionalen Aspekten des Handelns. Für ein konzeptionelles Verständnis des Zusammenspiels von Emotionen und Handeln greife ich darum auf Erkenntnisse der Emotionsforschung zurück. Ich beziehe mich unter anderem auf verschiedene Überlegungen von Rainer Schützeichel (2012a, 2012b), der einen recht allgemeinen Ansatz für Emotions- und Handlungstheorien vorschlägt, mit dem nicht nur vielfältige Bezüge auf die unterschiedlichen Handlungssituationen von Planenden abgebildet, sondern auch speziell jene für Planung konstitutiven Diskrepanzen zwischen Ist und Soll in den Blick genommen werden können. Zudem weist er auf die spezifische Qualität der Intentionalität von Emotionen hin sowie auf die hohe Bedeutung irritierender Gefühle. Schützeichel schlägt eine Typisierung von Handlungsmodi vor, die auch für die planungstheoretische Diskussion fruchtbar gemacht werden kann. Schließlich bietet er mit dem Konzept der Emotionsmilieus einen Ansatz, mit dem die Zusammenhänge von Sozialstruktur und Emotionen beleuchtet werden können.[5]

\subsection{Affektive Intentionalität und emotionsbezogene Handlungsmodi}

In Belief-Desire-Theorien wird angenommen, dass es Konstellationsbündel im Zusammenspiel von Wünschen und Überzeugungen sind, die für Handeln respektive für das Zustandekommen und die Funktion von Gefühlen ausschlaggebend sind (Schützeichel 2012a: 228). Mit entsprechender Theoriebildung nimmt Schützeichel an, dass auch Emotionen aus Vergleichsprozessen resultieren:

„Vor dem Hintergrund und auf der Basis von Wahrnehmungen, Einschätzungen und Meinungen artikulieren Emotionen in ihrer Sprache, dem Fühlen, ob reale oder imaginierte Zustände oder Ereignisse den ,desires', Präferenzen oder Prioritäten eines erlebenden und handelnden Akteurs entsprechen und ob die mögliche Diskrepanz 
zwischen ,desires' und Zuständen oder Ereignissen durch handelnde Interventionen verändert werden muss.“(Schützeichel 2012a: 238)[6]

Demnach vermögen Emotionen Handelnde negativ oder positiv an Situationen zu binden. Dabei kommt dem Fühlen eine funktionale und dabei intentionale Rolle zu, weil es dazu anleitet, Überzeugungen, Situationen oder Wünsche zu überprüfen, um sie beizubehalten oder zu verändern (ebd.). Schützeichel unterscheidet verschiedene Arten von Überzeugungen (beliefs): orientierende in Bezug auf Situationsdefinitionen, evaluative hinsichtlich Situationsbewertungen, instrumentelle bezüglich der Geeignetheit von Mitteln sowie normative Überzeugungen im Hinblick auf die Angemessenheit von Handlungszielen und Handlungsfolgen (ebd.: 241). Hier ist eine hohe Übertragbarkeit auf jene Wissensformen gegeben, die gemäß Rittel an Planung beteiligt sind. Dass sich Schützeichel dabei auf Überzeugungen und nicht auf Wissen stützt, scheint der Übertragbarkeit keinen Abbruch zu tun. Im Gegenteil schwingt in ,Überzeugung' schon mit, dass es nicht um eindeutiges Wissen gehen kann, genauso wie Rittel - das zeigt sein Begriff der Bösartigkeit - nicht in jedem Fall von der Möglichkeit eindeutigen Wissens ausgeht. Welche Wünsche (desires) sind nun konzeptionell bei der Entstehung von Emotionen zu berücksichtigen? Schützeichel (2012b: 476 f.) fasst die Kategorie der desires bewusst sehr breit: Sie reichen von „normative[n] Erwartungen“ bis hin zu „körperliche[n] Habitualisierungen“ und beziehen sich „auf zu realisierende oder zu vermeidende Zustände“. Insbesondere können so jene soziologisch bedeutsamen Wunschdimensionen abgebildet werden, die sich auf die physisch-leibliche Integrität beziehen, auf soziale Kontexte mit deren materiellen und symbolischen Dimensionen (wie Identitäts- und Anerkennungsansprüche) sowie auf die Geltung normativer Ordnungen in sozialen Konstellationen (ebd.: 477 ff., Schützeichel 2012a: 240 ff.). Auch wunschseitig scheint damit das Belief-Desires-Modell an das Handeln von Planenden anschlussfähig. Nicht zuletzt kann auch machtvermittelt-strategisches Handeln durch seine möglichen Sanktionen (auf physischer, materieller oder symbolischer Ebene) dargestellt werden.

Mit Schützeichel (2012a: 229) gehe ich von einer spezifischen Qualität von Gefühlen aus, davon, dass sie einen intentionalen Gehalt haben, dass sie „sich uns in einem intentionalen Fühlen mit[teilen]“. Es geht also weder um eine emotionale Bewertung des Wahrnehmens selbst (wie in evaluativkognitiven Theorien) noch um ein direktes Empfinden eines Zustandes an sich (wie in klassischen Gefühlstheorien). Vielmehr ist mit dem emotionalen Belief-Desire-Ansatz von einer abgleichend-affektiven Bewertung eines Zustandes in Bezug auf eigene Wünsche, das heißt von einem intentionalen Empfinden, auszugehen. Dabei kann von einer „affektiven Intentionalität“ gesprochen werden, weil durch die affektive Bezugnahme auf eine Intention diese selbst zu einer affektiv gefärbten respektive bedeutsamen wird (ebd.; vgl. auch Rothermund/Eder 2011: 166). Schützeichel bezieht sich unter anderem auf Jan Slaby (2011: 37), der Gefühle und Emotionen beschreibt „als verhaltensartig verfasste Vollzüge - als ein Orientiert- und Situiertsein in der Welt, als eng verschränkt mit dem Handeln und Tätigsein der fühlenden Person“. 
Abb. 1 Typologie verschiedener Handlungsmodi (Quelle: Schützeichel 2012a: 242 ff., 2012b: 476; Abbildung modifiziert und ergänzt)
Nun ist die affektive Intentionalität durchaus folgenreich für das Verständnis von strategisch-zielgerichtetem Planungshandeln als einem teleologischen, das heißt auf einen Zweck hin ausgerichtetem Handeln. Denn wenn eine emotionale Grundierung von Intentionen anzunehmen ist, wenn Gefühle „auf dynamische Weise den Bereich des für eine Person konkret und real Möglichen ab[stecken]“ (ebd.: 33), dann gilt es auch die emotionalen Aspekte teleologischen Handelns anzuerkennen. Damit ist die Zweckgerichtetheit des Handelns von Planenden nicht länger als eine rein zweckrationale, sondern auch als eine emotionale zu begreifen. Der strategisch von Planenden verfolgte Zweck liegt also nicht nur im Außen vermeintlich objektiver Projektziele. Der Möglichkeitsraum der Zwecke selbst wird mit dem Kriterium der subjektiv-emotionalen Akzeptabilität vielmehr vorstrukturiert. Emotional nicht akzeptable Zwecke werden kaum die Chance haben, zu handlungsleitenden Motiven zu werden: „Denn Menschen deuten ihre Lebenssituation [...] nicht nur so, wie sie sie sehen, sondern auch so, wie sie es ,aushalten“ können“ (Arnold 2005: 249). Dabei muss der Einfluss von Emotionen auf Handlungen als ein indirekter verstanden werden, da stets über beliefs und desires vermittelt. Teleologisches Handeln muss also nicht als ein - im Sinne einer emotionalen Determination - naturalistisches verstanden werden. Gleichwohl ist es aber nicht frei vom Empfinden der Akteure (vgl. Schützeichel 2012a: 238 f.).

Um das Verhältnis zwischen Emotion und Handlung differenzierter zu beschreiben, unterscheidet Schützeichel bezüglich der Bewertung von möglichen emotionalen Diskrepanzerfahrungen zwischen beliefs und desires die in Abbildung 1 dargestellten verschiedenen Handlungsmodi.

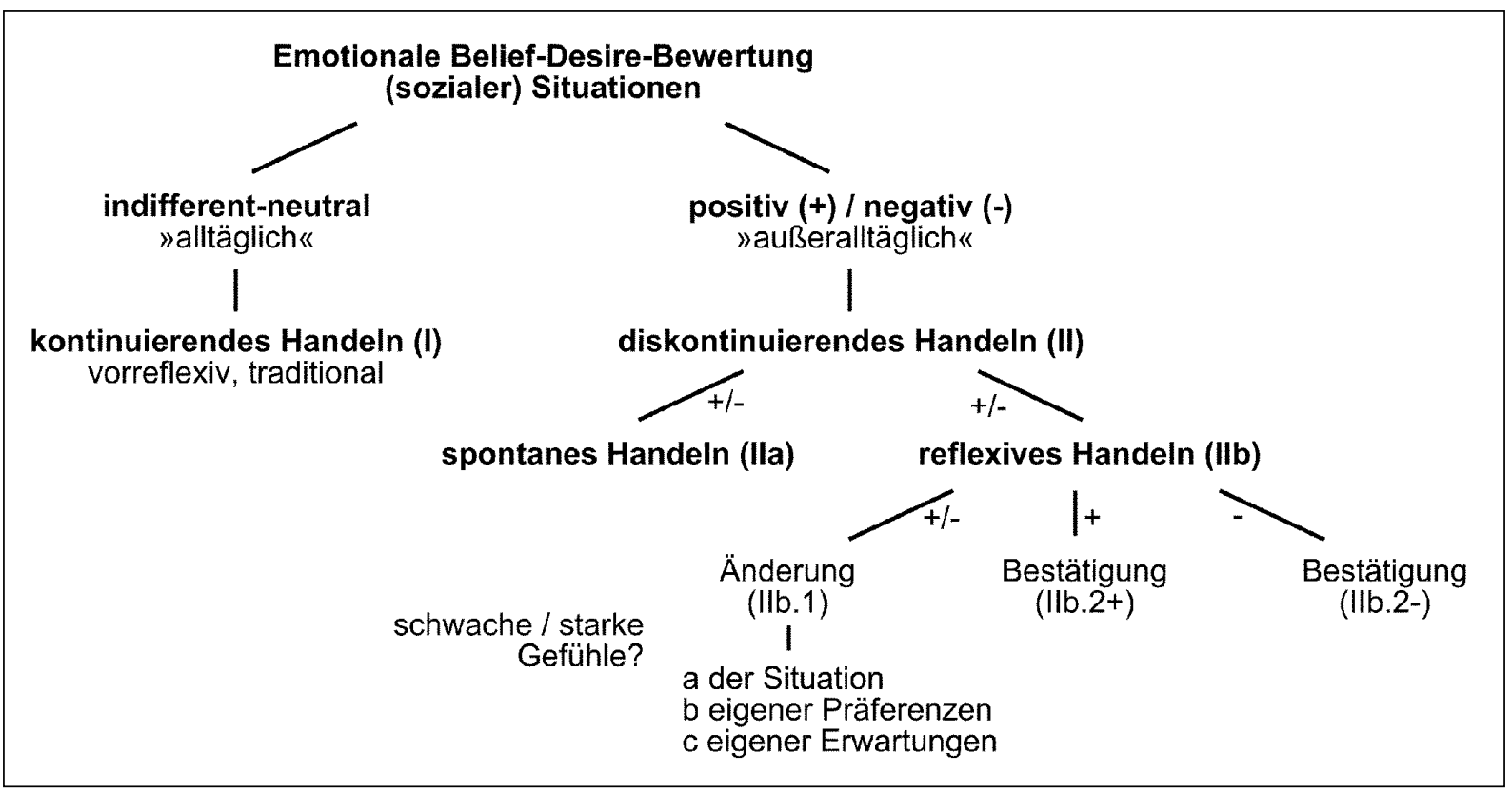

Schützeichel kennzeichnet diese folgendermaßen (2012a: 242 f.): Als kontinuierendes Handeln (I), das durch Gefühle geprägt ist, die nur schwach vernehmbar sind (d.h. eine niedrige Valenz haben), wie ein Fühlen von Ruhe, Zufriedenheit oder Gelassenheit. Sie führen zur Beibehaltung des Belief-Desire-Zustands und kennzeichnen Handeln als „alltäglich“, „vorreflexiv“, „normal“ oder „traditional“, als eines, das kontinuiert werden kann. Diskontinuierendes Handeln (II) ist „außeralltäglich“ und geht mit 
positiven oder negativen Gefühlen von hoher Valenz einher. Hier kann weiter unterschieden werden in spontanes Handeln (IIa), das „vorreflexiv“ und „gegenwartsbezogen“ ist, sowie in reflexives Handeln (IIb), das „zukunftsbezogen“ ist. Letzteres kann - mit ganz unterschiedlichen Konsequenzen für das intentionale Belief-Desire-System - in zwei weitere Typen differenziert werden: Im Typ Änderung (IIb.1) wird die emotionale Differenzerfahrung negativ empfunden, womit zur Reflexion angeleitet wird: Ist (a) die Situation zu verändern, ist (b) die eigene Präferenz auf das, was ist, zu verändern, oder ist (c) die eigene Erwartung auf das, was sein soll, zu verändern? Beim zweiten Typ Bestätigung (IIb.2+) handelt es sich um eine positive erfahrene Diskrepanz zwischen Situation und Erwartung, die ebenfalls zu einer Reflexivisierung des Handelns führt, nun aber in einem bestätigenden Sinne, das heißt ohne Anpassungen an das Belief-Desire-System. Eine weitere Differenzierung, die Schützeichel nicht aufführt, scheint mir geboten: Denn auch der Bestätigungs-Typ (IIb.2) kann mit einer negativen Erfahrung hinterlegt sein, und zwar dann, wenn im Falle negativ-reflexiver Differenzerfahrungen keine der Veränderungsoptionen des Typs IIb.1 subjektiv plausibel (z.B. durch gefestigte Überzeugungen) oder möglich sind (z.B. durch mittelbar nicht auflösbare Zwänge). Dann kann eine gegebene Situation zu wiederholten respektive anhaltenden Leidenserfahrungen führen - eine Erfahrung, die unter anderem in Bezug auf strukturell verfestigte Situationen, die von politischen Akteuren dennoch adressiert werden, von Relevanz ist.[7]

\subsection{Verunsicherung und,emotionale Rationalität}

Emotionen aktivieren nicht nur verschiedene Handlungsmodi. Vielmehr spielen sie in alle Handlungssituationen hinein, da diese „nicht nur kognitiv definiert, sondern auch affektiv bewertet“ werden (Schützeichel 2012a: 227). Gerhard Roth (2009: 175) stellt dabei aus neurobiologischer Sicht auf einen engen Zusammenhang zwischen Handeln und Gefühl ab, weil „alles, was Vernunft und Verstand als Ratschläge erteilen, für den, der die eigentliche Handlungsentscheidung trifft, emotional akzeptabel sein muss“. Rittels erkenntnistheoretischer Schluss, dass am Ende einer Kette verstandesmäßigen Abwägens ein Spontanurteil steht, wird von Roth qualifiziert, indem er davon ausgeht, dass es „kein rein rationales Handeln [gibt]. Am Ende eines noch so langen Prozesses des Abwägens steht immer ein emotionales Für oder Wider" (ebd., Hervorh. i. Orig.) Weiter schließt Roth, dass es „die Chance der Vernunft ist [...], mögliche Konsequenzen unserer Handlungen so aufzuzeigen, dass damit starke Gefühle verbunden sind, denn nur durch sie kann unser Verhalten geändert werden“ (ebd.). Es gilt also nicht nur die herausgehobene Rolle von Gefühlen als Instanz der Urteilsbildung zu berücksichtigen, sondern auch die besondere Bedeutung von starken Gefühlen. Entsprechend wird in der pragmatistischen Emotionsforschung auf die Wesentlichkeit von „einschneidenden emotionalen Erfahrungen“ verwiesen, wenn Wandlungsprozesse an subjektiv tief verankerte Bewertungskriterien heranreichen sollen (Pettenkofer 2012: 204, mit Bezug auf James). Übertragen auf Schützeichels Handlungsmodi wäre damit zu erwarten, dass sich auch in jenem reflexiven Handlungsmodus 
- der auf Änderung zielt (IIb.1) - Veränderungen von grundlegenderen persönlichen Präferenzen (b) oder Zielen (c) nicht ohne Weiteres ergeben, sondern nur bei Anwesenheit von tiefen Emotionen.

Emotionen wird in diesem Zusammenhang eine konstitutive Rolle bezüglich Rationalität und Reflexivität zugesprochen (Schützeichel 2012a: 232, 251). Dabei kommt speziell den Momenten von Irritation und Beunruhigung eine besondere Funktion nicht nur bei der Bewertung im Rahmen von Reflexionsprozessen zu, sondern auch als deren Voraussetzung. So können emotional irritierende Situationen als erforderlich für Reflexivität verstanden werden, wobei die einsetzende Reflexion nicht an der eigentlichen Situation haltmachen muss, sondern, ,verallgemeinernd die jeweilige Gesellschaftsordnung insgesamt als problematisch erscheinen lassen kann“ (Pettenkofer 2012: 212, 203).

Im Umkehrschluss werden geringe Rechtfertigungsniveaus gerade dann gewählt, wenn keine „Unruhe“ aufkommen soll (Boltanski 2010: 99). Seitens der neurowissenschaftlichen politischen Psychologie wird betont, dass „Ängstlichkeit im Sinne von Beunruhigtsein [...] als emotionale Bedingung [erscheint], um Rationalität zu entfalten, Gewohnheiten zu überwinden und die Mühe der Informationsbeschaffung zu alternativen Handlungsweisen auf sich zu nehmen. Emotionalität steht damit im Dienste des Lernens“ (Nullmeier 2006: 93). Analog geht es auch emotionspädagogischer Erwachsenenbildung darum, Angst nicht zu vermeiden. Arnolds Konzept des Emotionslernens gründet gerade in der Phase der „Verunsicherung“, gefolgt von jenen der „Information“ und "Transformation“ (Arnold 2005: 123 ff., $229 \mathrm{ff.}$., 253).

Der Erkenntnis, dass Emotionen, Handeln und Urteilsbildung unauflösbar aufeinander bezogen sind, wurde in den letzten Jahrzehnten auch insofern Rechnung getragen, als diesbezüglich revidierte Konzepte von Rationalität vorgeschlagen wurden. Ciompi führte 1982 den Begriff der „Affektlogik“ ein (2005: 31), Meier-Seethaler schlug 1997 ein Konzept der „emotionalen Vernunft“ vor (2001: 249), de Sousa im selben Jahr eines der „rationalen Emotionalität“ (ebd.). Der Einfachheit halber werde ich im Folgenden von ,emotionaler Rationalität' sprechen und meine mit MeierSeethaler damit „einen umfassenden Begriff der Vernunft [...], der gleichermaßen rationale wie emotionale Anteile enthält“ (ebd.). Ich grenze mich dabei von einem Verständnis von emotionaler Intelligenz ab, bei dem in Bezug auf Daniel Goleman Gefühle für persönlichen Erfolg instrumentalisiert werden (vgl. ebd.). Auch ziele ich nicht auf die Frage der (Un-) Angemessenheit von Gefühlsreaktionen.

\subsection{Eingebettetsein in Emotionsmilieus}

In einer Arbeit über soziale Bewegungen untersucht Armin Stickler, welche Wirkungen Institutionalisierungen von sozialen Bewegungen haben. Dabei nimmt er an - womit er die Aussagen des vorherigen Abschnitts bestätigt -, dass es bezüglich gesellschaftlicher Veränderung um die Frage geht: „Wann wird Gesellschaft irritiert und wann nicht“? (Stickler 2011: 132). Er stellt dar, dass Institutionen das Vermögen von Akteuren strukturieren, Dinge wahrzunehmen und Wirklichkeit zu 
konstruieren, wobei gleichzeitig - durch Vernetzung, Kommunikation und organisatorische Formalisierung - soziale Praxen vereinheitlicht werden und damit Bewegungsorganisationen letztlich „als Normalisierer von Irritationen“ (ebd.: 131, 126) auftreten. Stickler hält skeptisch fest, dass sowohl von der Art der Organisation als auch von den „konkreten Verflechtungen mit der diskursiv-symbolischen Umwelt“ (ebd.: 132) Beschränkungen ausgehen, denen nicht willentlich entkommen werden kann. Dies kann mit Erkenntnissen der Institutionenpsychologie bestätigt werden, in der empirisch begründet angenommen wird, dass „Institutionen [...] jene Gefühle erzeugen [können], die die Kontinuität und Stabilität der erzeugenden Institution sicherstellen“ (Nullmeier 2006: 96).

Mit Schützeichels Erklärungsansatz von Emotionsmilieus können solche Institutioneneffekte emotionstheoretisch konzeptualisiert werden. Schützeichel vertritt die These, dass (kommunikative) Milieus auch in emotionssoziologischer Hinsicht eine besondere Relevanz haben. Unter Milieus versteht er mit Bezug auf G. Schulze keine „handlungsmächtigen sozialen Einheiten“ oder Gruppen, sondern Personenkonstellationen, die sich durch eine „erhöhte Binnenkommunikation voneinander abheben“, Netzwerke, die sich ihren Angehörigen als gemeinsame „Resonanzböden“ darstellen und die in Bezug auf ihre „Erfahrungsräume“ ähnlich sind (Schützeichel 2012b: 48o f.). Als „Emotionsmilieus“ kommt diesen Netzwerken mit ihren Orten „kommunikativer Verdichtung“ eine besondere Bedeutung zu, da in ihnen fortwährend desires und beliefs im Rahmen „kommunikativer Bestätigungen" abgeglichen werden - in einem Prozess, der sich wiederum durch „emotionale Gratifikationen“ auszeichnet (ebd.: 482). Darum vertritt Schützeichel die These, dass soziale Konstellationen auch in Bezug darauf gewünscht, bevorzugt und ausgewählt werden, ob in ihnen die eigenen Wünsche wertgeschätzt werden oder nicht. Für ihn beruhen selbst die „sozialen Grenzen von Milieus [...] auf affektiven Grenzziehungen“ (ebd.).

Mit dem Ansatz, dass in Netzwerken und in Abhängigkeit von Dichte und Nähe der Kommunizierenden sich emotionsvermittelt Angleichungen bezüglich der sich herausbildenden Belief-Desire-Strukturen oder gar personelle Ausdifferenzierungen ergeben, gibt Schützeichel ein Argumentarium an die Hand, Akteurskonstellationen unter dem Aspekt des Gefühlshaushalts ihrer Mitglieder zu analysieren. Er fokussiert damit auf die emotionale Dimension von intersubjektiven und gruppenbezogenen Anerkennungsprozessen, denen im Verständnis von Axel Honneth Wertezuschreibungen zugrunde liegen. Mit Honneth (1992: 205 ff., 2010: 261 ff.) geht es bei Anerkennungsprozessen letztlich darum, ,sich in seinen eigenen Leistungen und Fähigkeiten als wertvoll für die Gesellschaft zu erfahren".

Mit Emotionsmilieus soziale Grenzziehungen zu erklären, scheint mir dort, wo die Netzwerkzugehörigkeit von einem hohen Grad von Freiwilligkeit geprägt ist, nicht unplausibel - hingegen dürfte mit zunehmenden Abhängigkeiten (z.B. durch berufliche oder private Bindungen an Einrichtungen oder Orte) auch die Erklärungskraft des Ansatzes abnehmen. Dennoch denke ich, dass genau in Bezug auf durch Emotionsmilieus stabilisierte Formen wiederholt gleicher oder ähnlicher Wertezuschreibungen eine Diskussion des Innovationsgehalts von Netzwerken ansetzen könnte, wie 
auch eine Kritik der „Eigenlogik der Städte“(Löw 2008); eine Kritik, die dabei jenseits lokalistischer Handlungszuschreibungen das Vorhandensein von spezifischen Emotionsmilieus in den Blick nimmt. Solche Emotionsmilieus wären weder geographisch verengt noch stadtzentristisch zu begreifen und dennoch würden in ihnen Städte als Orte kommunikativer Verdichtung über entsprechende Netzwerkstrukturen Niederschlag finden. In solchen Milieus wären bezüglich der herrschenden Meinung abweichende oder politisch dissidente Ansichten zwar weiterhin möglich, aber zugleich mit (besonders) hohen und wiederkehrenden emotionalen Unlust- und Frustmomenten verbunden und damit tendenziell erschwert. Da die Struktur kommunikativer Milieus als „Netzwerke von Netzwerken“ (Schützeichel 2012b: 481) verstanden werden kann, könnte über die Einschreibung dieser Netzwerke in räumliche Strukturen ein konzeptioneller Link zur Einschreibung von Haltungen und Überzeugungen in die analogen räumlichen Strukturen hergestellt werden. Insofern Handlungen nicht von Emotionen determiniert werden, wäre solch eine Kritik jedenfalls jenseits handlungsdeterminierender Überlegungen zu führen. Damit ginge es entgegen Löw also auch nicht um eine Logik des Ortes, sondern sehr wohl um eine auf die „individuellen Handlungen rückführbare Realität“ (Löw 2008: 77). Handeln behielte somit seinen politischen Charakter.

\subsection{Zur Schwierigkeit der Erforschung emotionaler Handlungsmotive}

Bei aller Bedeutung von Emotionen im Planungshandeln ist zu erwarten, dass sie schwer zu erforschen sind. Nicht zuletzt, weil anzunehmen ist, dass der Einfluss von Emotionen auf Entscheidungen oft „subtil“ bleibt und selbst für Handelnde wenig offensichtlich ist (Rothermund/Eder 2011: 179). Doch nicht nur Aussagen über die jeweilige Wirkmächtigkeit von Emotionen dürften schwierig sein, auch die quantitative Erforschung von Gefühlen sieht sich prinzipiellen Schwierigkeiten gegenüber. Denn beim Fühlen kommen jeweils subjektive Bewertungsmaßstäbe zur Geltung, und so muss dasselbe Ereignis vor dem Hintergrund unterschiedlicher intentionaler Belief-Desire-Strukturen zu unterschiedlichen Gefühlen führen.

Wer sollte schließlich für die Erforschung der emotionalen Beweggründe von Planenden geeignet sein? Sind es die Planer_innen selbst, sind es Sozialwissenschaftler_innen oder Psychoanalytiker_innen? Planende haben womöglich gar kein Interesse, ihre emotionalen Motive - das heißt letztlich ihre beliefs und desires - aus dem Bereich des unterbewusst Vorreflexiven zu holen -, sobald sie sich einmal im Ungefähren bezüglich ihrer Beiträge vergewissert haben, die sie zur,Verbesserung ' der von ihnen je spezifisch wahrgenommenen Welt und zur diesbezüglichen Übernahme von Verantwortung zu leisten bereit sind. (Ich werde darauf zurückkommen, dass gerade Emotionslernen zum Ziel hat, durch den Prozess des Umfühlens Veränderung der eigenen Weltsicht zu ermöglichen.)

Zumindest drängt der Arbeitsalltag nicht nach dem reflexiven Handlungsmodus der Veränderung von Präferenzen oder Überzeugungen, der durch eine Erforschung der Gefühlslagen aber durchaus droht, aktiviert zu werden. Auch Sozialwissenschaftler_innen wird es daher nicht leichtfallen, gerade über jene emotionalen Beweggründe von Planenden zu erfahren, 
die besonders intim oder persönlich grundlegend sind. Zudem kann angenommen werden, dass es Wissenschaftler_innen grundsätzlich schwerfallen muss, spezifische Dilemmata in der Planung selbst nachzufühlen, insofern Planung unter anderem mit ihrem spezifischen Praxisvollzug im Fluss der Zeit und ihrem darin eingelagerten faktischen Setzen und Ausschließen von Alternativen nicht von derselben „scholastischen Sicht“ gekennzeichnet ist wie Wissenschaft (Bourdieu 1998: 207 ff., 1993: 149 ff.). Bliebe zum Ergründen der emotionalen Beweggründe die Psychoanalyse: Doch warum sollten Planende aus Forschungsinteresse Lust daran haben, ihre Planungspraxis mit der Couch zu tauschen?[8]

\section{Planungstheorie und Emotionen: Planungsansätze einer, dritten Generation'}

Der erweiterte, das Emotionale einbeziehende Vernunftbegriff erfordert meines Erachtens eine angemessene planungstheoretische Berücksichtigung von Gefühlen. Ich möchte die These aufstellen, dass genau das konzeptionelle Ausblenden der emotionalen Dimension bisherige Planungstheorien weitestgehend eint. Dies kann zumindest mit einem Blick auf die jüngere deutschsprachige Diskussion bestätigt werden. Der emotional turn scheint hier noch nicht angekommen.[9]

Um einen Beitrag zu leisten, diese konzeptionelle Leerstelle auszuweisen, schlage ich in Weiterentwicklung der bei Rittel (1972) ausgebreiteten Planungsansätze der „ersten und zweiten Generation“ vor, von Planungsansätzen einer ,dritten Generation' zu sprechen, insofern sie dem Aspekt der emotionalen Rationalität angemessen Rechnung tragen. Der Ausdruck der „dritten Generation“ stammt dabei von Walter Schönwandt (1999, 2002, 2011), der damit seinerseits eine planungstheoretische Weiterentwicklung der ersten beiden Generationen beansprucht hat. Ich möchte mich von seinem Konzept jedoch klar distanzieren. Auch Schönwandt vermeidet es, Emotionen weitergehend und konzeptionell zu berücksichtigen. Er zielt vor allem darauf, unter Berücksichtigung diverser Denkfallen und unter der Forderung „Probleme zuerst“ (Schönwandt 2011: 292) ein klares Verständnis von Sachlage und verwendeten Konstrukten (Begrifflichkeiten, Konzepten etc.) im Rahmen von Planungsprozessen herzustellen. Was theoretisch plausibel erscheint, planungslogisch im Sinne einer technischwissenschaftlichen Rationalität geboten ist und planungspraktisch zu mehr Eindeutigkeit führen soll, erinnert aber zugleich daran, letztlich (weitgehend) ideale Diskursbedingungen anzunehmen und verstandesrational handeln zu wollen und zu können. So gesehen oszilliert Schönwandts ,dritte Generation' methodisch zwischen den Ansätzen der ersten und zweiten Generation, ohne im eigentlichen Sinne falsch zu sein, aber auch ohne über sie hinauszuweisen. Insbesondere handelt es sich dabei nicht um eine Weiterentwicklung des der Planung zugrunde liegenden Rationalitäts- und Erkenntnismodells. Dies war es jedoch, was Rittel erst zu seinem paradigmatischen Schritt veranlasste, eine zweite Generation einzuführen. Und daher schlage ich auch nicht in Anschluss an Schönwandt eine vierte, sondern in Anschluss an Rittel und an Reuter, der die zweite Generation bezüglich Fragen des Machtgebrauchs qualifizierte, eine, dritte Generation“ vor. 


\subsection{Umgang mit Widersprüchlichkeit und Unsicherheit: Bisherige Konzeptionen}

Bevor ich näher auf Planungsansätze einer ,dritten Generation“ eingehe, möchte ich anhand von zwei jüngeren Ansätzen diskutieren, wie Unsicherheiten, Uneindeutigkeiten, Zweifel, Widersprüchlichkeiten etc. in Planungstheorien Eingang gefunden haben. Beide beinhalten einen eigenen Erklärungsanspruch für Planungshandeln: Siebel postuliert eine eigene Planungsrationalität, Jessen und Reuter zielen auf Erfahrung.

Walter Siebel (2006: 206) stellt der technischen und politischen Rationalität in der Planung eine dritte ökologische ergänzend zur Seite, die auf den „Grad der Revidierbarkeit des Geplanten“ zielt. Er bezieht diese einerseits auf das von Jessen formulierte Prinzip des „prinzipiellen Irrtumsvorbehalts“ (ebd.: 204) - wonach Fehler korrigierbar sein sollten -, andererseits auf die „Irrationalitäten politischer Willensbildung“(ebd.: 206) - die darin lägen, dass Interessen künftiger Generationen heute nur schwerlich antizipiert werden könnten. Da das gleichzeitige Nebeneinander dieser Rationalitäten zu Widersprüchen führe, schlägt Siebel für Planung eine vierte Rationalität vor, die darin besteht, „die Widersprüche zwischen den Anforderungen der übrigen drei auszuhalten“ und dazu befähigt, ,zwischen widersprüchlichen Aufgaben zu lavieren“ (ebd.: $208 \mathrm{f}$.).

Nun ist die ökologische Rationalität jedoch zu kritisieren: Denn Entscheidungen in Bezug auf die Korrekturfreundlichkeit von Fehlern oder auf ,künftige' Interessen sind nichts anderes als heutige, normativ-strategische und damit letztlich politische Entscheide. Sie gehen voll in der politischen Rationalität auf. Bei der ökologischen Rationalität handelt es sich also vielmehr um Zuschreibungen vermeintlicher Zielsetzungen in ein Ökologisches, nicht aber um sich aus dieser Rationalität selbst ergebende oder notwendig ableitbare Kriterien. Damit werden politische Entscheide über eine zukunftsbezogene Gestaltung gesellschaftlicher Naturverhältnisse jedoch unzulässig naturalisiert (vgl. Swyngedouw 2009). Was aber bleibt dann von der „eigentlichen Rationalität der Planung“? (Siebel 2006: 209). Ist es sinnvoll, das Vermögen, Widersprüche auszuhalten, mittels einer spezifischen Rationalität zu ehren? Werden sie dadurch nicht vorschnell als unveränderbar erachtet, anstatt ihre Entstehungsbedingungen einer Analyse und politischen Bearbeitbarkeit zugänglich zu machen? Zweifelsohne gehört heute ein Umgang mit widersprüchlichen Anforderungen zur Planung aber eben auch zur Politik. Sie jedoch in einer Planungsrationalität zum Verschwinden zu bringen und damit der politischen Verhandlung konzeptuell-theoretisch zu entziehen, stellt ein depolitisierendes Moment dar. Überzeugender wäre es, hier die Frage zu stellen, was genau ein Aushaltenkönnen von Widersprüchen bedingt, wozu dieses führen könnte, wie der Umgang mit ihnen ganz konkret möglich und konzeptualisierbar wird und welche Kompetenzen Planende dabei haben müssten.

Hinsichtlich solcher Kompetenzen ziehe ich nun einen Beitrag von Johann Jessen und Wolf D. Reuter (2006) heran. Rittel (1972: 54 ff.) sah im Rückgriff auf Erfahrung eine Option, angesichts von (erforderlichen) Spontanurteilen dennoch routiniert handeln zu können. Daran knüpfen Jessen und Reuter an. Sie konzipieren Erfahrung als Wissen, auf das in 
unterschiedlichen, von zum Beispiel von Unordnung, Wertediversität, Konflikten oder Widersprüchen gekennzeichneten Situationen zurückgegriffen werden kann. Sie qualifizieren damit die bei Siebel nur postulierte Planungsrationalität, indem sie eine Antwort auf die Frage geben, was Planende befähigt, mit Widersprüchlichkeit erfolgreich umzugehen - nämlich Erfahrung. Als Quell erfahrenen Handelns verweisen sie auf eine „Verschmelzung von Wissen, normativer Orientierung und Intuition" (Jessen/Reuter 2006: 53). Sie greifen dabei auf das aristotelische Konzept der „Phronesis“ zurück, das auf Gemeinwohlorientierung statt Eigeninteresse fokussiert, sowie auf „eine aus der Praxis heraus [...] erworbene Klugheit“ (ebd.: 54). Dabei stellen sie Erfahrung weitgehend in den Dienst von situationsspezifischer, aber dennoch strategischer Intention und erfolgreicher Praxis (ebd.: 42 ff., 54 ff.).

Bezogen auf Schützeichels Handlungsmodi kann Erfahrungshandeln vorreflexives Handeln sein (I, IIa), in Situationen jedoch, die zur stärkeren Reflexion anleiten, kann Erfahrungswissen dazu beitragen, auch in irritierenden Situationen noch recht routiniert Lösungswege auszumachen - das heißt, die Situation (IIb.1a) erfolgreich zu verändern -, um im Ergebnis rasch zu einer neuen Situationsbewertung vom Typ positiv-emotionaler Bestätigung (IIb.2+) zu gelangen. Als wesentliche Eigenschaft des Handelns unter Rückgriff auf Erfahrung wird gerade gelten müssen, dass es trotz potenzieller Irritation nicht zu Änderungen an den eigenen intentionalen Belief-Desire-Strukturen (im Sinne IIb.1) kommt. Könnte es daher nicht sein, dass gerade die planerische Kernkompetenz der Erfahrung dazu beiträgt, die Veränderung von eigenen Überzeugungen und Wünschen, von alternativen Formen des Aushandelns von Werten oder einem andern Umgang mit Dissens etc. zu marginalisieren? Das hieße, dass Erfahrung auch dazu beitragen könnte, in einem orthodoxen Sinne bestehende Strukturen zu stabilisieren, gerade dadurch, dass mit Unsicherheiten routiniert umgegangen wird.

In der planungstheoretischen Konzeption von Jessen und Reuter sehe ich zwar einen empirisch hochplausiblen Erklärungsansatz, doch werden mit ihm politisch relevante Fragen zugleich konzeptuell verdeckt. Gerade dort, wo Erfahrung Planende befähigt, Lösungen vorzuschlagen, die gesellschaftlich „funktionieren“ und die damit ein weitgehend routiniertes Handeln ermöglichen, rückt sie diese Qualifikation in die Nähe der von Žižek beschriebenen „Post-Politik“ (2009: 36). Ein solch potenziell depolitisierendes Moment gelangt auf doppeltem Weg in die Konzeption bei Jessen und Reuter. Zum einen wird durch den Fokus auf den Gemeinwohlbezug der Blick auf die Subjektivität von Intentionen und deren Affektionalität verstellt. Damit wird unter anderem jener Quell von Kritik unsichtbar gemacht, den Boltanski (2010: 130 ff.) mit dem „hermeneutischen Widerspruch" beschreibt und bezüglich dessen Bourdieu (1998: 225) von den „Kosten für den Verschleierungsaufwand“ des Auseinanderfallens von eigener und offizieller Position spricht. Bourdieu weist daher neben Erfahrung noch weitere Handlungsmotivationen sozialer Akteure aus (Bourdieu/Wacquant 1996: 170). Zum anderen konzipieren Jessen und Reuter (2006: 54) die „soziale Umgebung, [...] eine Stadt, ein Amt“- und damit auch jene Akteure, durch die Erfahrung und 
kluge Praxis bescheinigt werden - als ein „unabhängiges Kriterium“. Diese Unabhängigkeit scheint mir aber nicht haltbar. Einerseits nicht hinsichtlich institutionen- und gruppenbezogener Anerkennungsverhältnisse und Emotionsmilieus, da sich allein schon im Attestieren von kluger Praxis ein emotional vielfältig bedeutender Akt verbirgt.[10] Nicht nur setzen sich mit solch einem Attestieren die Subjekte der sozialen Umgebung in eine dezidierte Beziehung zur erfahrenen Planerautorität, vielmehr entlasten sie sich selbst von der Suche nach einer möglicherweise noch klügeren (politischen) Praxis. Andererseits wird durch diese postulierte Unabhängigkeit die soziale Umgebung konzeptionell homogenisiert. Dies kommt einem Akt einer ideellen Kollektivierung gleich, durch den politisch dissidente Minderheitenpositionen dem Blickfeld entzogen werden.

\subsection{Zur Diskussion gestellt: Planungsansätze einer ,dritten Generation“}

Im Lichte einer emotionalen Rationalität scheinen mir bestehende planungstheoretische Zugänge und Planungsstrategien erweiterungsbedürftig, weil ihnen unter anderem Handlungsmodelle zugrunde liegen, die hinsichtlich der affektiven Intentionalität nicht elaboriert genug sind. Zwar wird mit bestehender Theoriebildung anerkannt, dass Planende ihre eigenen Wertehaltungen in Planungsprozesse einbringen,[11] dies aber - wie mir scheint - mit einem anderen, vor allem normativ-strategischen Augenmerk. Solch eine Fokussierung dürfte daher rühren, dass als Gegenstände von Planung meist zukunftsbezogene Interessen und Ziele gelten oder pragmatistisch-situationsbezogene, die aber ihrerseits einen am Zweck der Planung orientierten Charakter haben. Es geht bislang also eher darum, dass auch den eigenen Überzeugungen und Wünschen zum Durchbruch verholfen wird. Es wird jedoch nicht beleuchtet, wie diese Überzeugungen zustande kommen, wie sie als emotionale Grundierung des Handelns erneuert werden, wie und in welchem Maße die erwähnten Momente der Selbstversicherung handlungsleitend sind, wie sich strategisch-normative Aushandlungsprozesse anfühlen oder welche emotionale Konsequenzen Produkte von Planung (Pläne, Programme, Leitbilder etc.) haben - um nur einige Fragen zu nennen. Doch gerade im Ernstnehmen solch emotionaler Dimensionen sehe ich eine wichtige politische Bedeutung. Wenn „eine emotional neutrale Staatstätigkeit schlicht unmöglich ist" und Bürger_innen ohnehin einem „Gefühlsmanagement“ (Heidenreich 2012: 21) ausgesetzt sind, dann müsste nicht zuletzt mit Blick auf die „Möglichkeit einer neuen, expliziten und transparenten Gefühlspolitik“ gefragt werden: „Welche Form politischer Steuerung von Gefühlen ist normativ begründbar?" (ebd., Hervorh. i. Orig.).

Um all solche Fragen systematischer und besser in den Blick zu bekommen, stelle ich hiermit Planungsansätze einer ,dritten Generation' zur Diskussion. Nicht weil entsprechende Ansätze schon allgegenwärtig sind (was sie nicht sind), sondern da mit Planung und dem Denken über Planung reflektiert werden müsste, wie die emotionale Dimension zu bearbeiten wäre, um mit ihr im Sinne einer politischen Verantwortung bewusst umgehen zu können. Selbstverständlich kann solch ein mit ,Verantwortung postuliertes, normatives Moment durch Machthandeln gebrochen werden. 


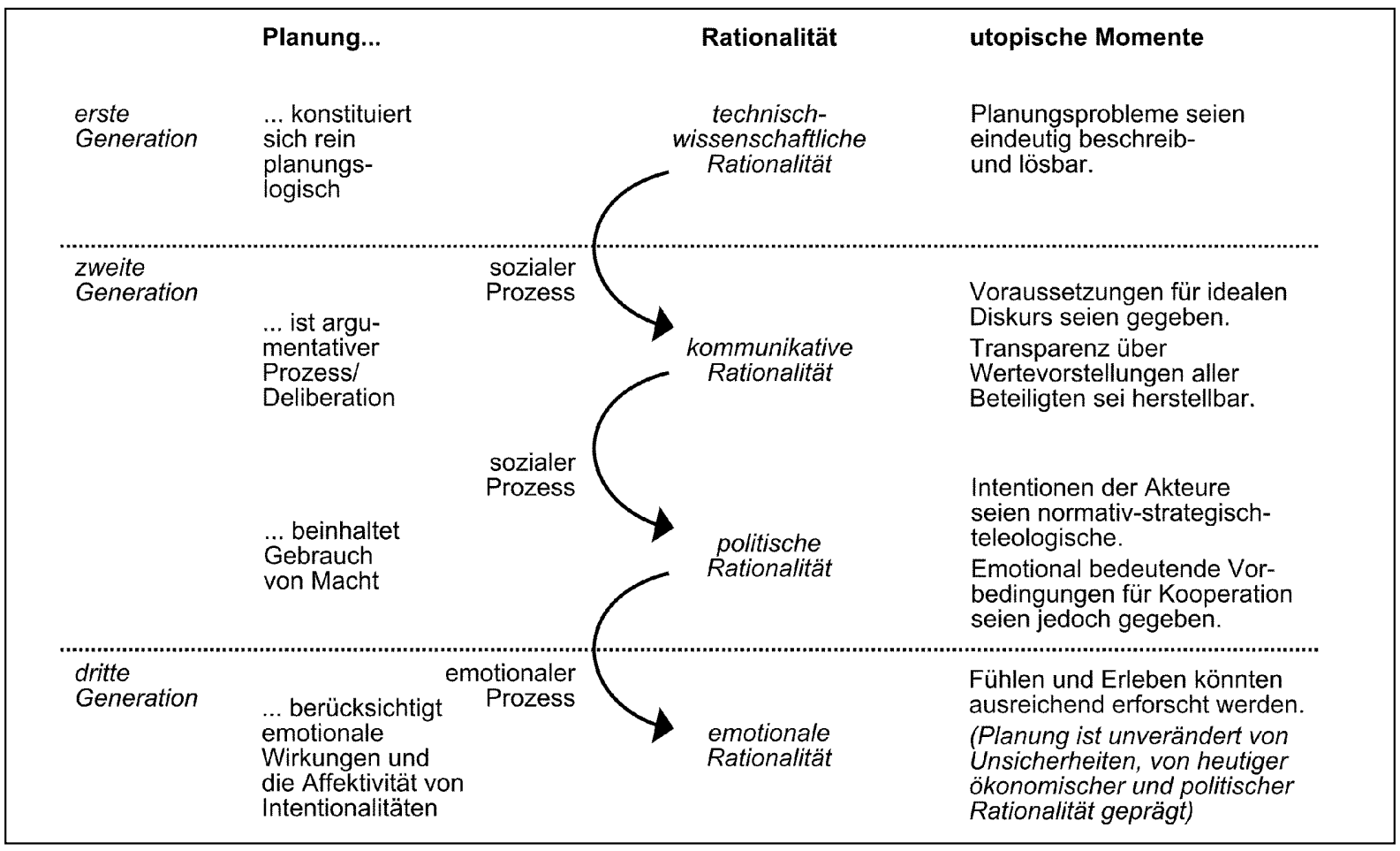

Die ,politische Rationalität‘ wird durch die emotionale nicht aufgehoben, sie geht vielmehr in ihr auf, da jedes politisches Handeln gleichsam eine emotionale Dimension hat. Zur Klarstellung: Ich möchte hiermit weder einem wie auch immer gearteten emotionalen Handlungsdeterminismus das Wort reden (was emotionstheoretisch ohnehin schlecht begründbar wäre), Abb. 2 Erweiterung der Rationalitätsverständnisse in der Planung (Quelle: eigene Darstellung) noch gehe ich davon aus, dass eine emotionale Rationalität die ,bessere' sei (was noch keine Aussage über den Stellenwert von Emotionen beinhaltet). Es geht vielmehr um deskriptive wie normativ-strategische Zugänge zu Planung, mit denen im Lichte einer emotionalen Rationalität besser lesbar gemacht werden kann, wie Planende handeln, wie Planungen zustande kommen und was sie zu bewirken vermögen. Die Ansätze sollen also sowohl theoretische Zugänge als auch konkrete Planungsstrategien umfassen.

Mit der Gliederung in Abbildung 2 fasse ich die Paradigmen der kommunikativen und politischen Rationalität im Rahmen der ,zweiten Generation' zusammen, da beide in der sozialen Vermittlung planerischen Tuns wurzeln.[12] Eine ökonomische Rationalität weise ich nicht gesondert aus, obwohl auch mit ihr eine plausible Weiterentwicklung bisheriger Ansätze denkbar wäre. Nicht nur, da ihr eine eminent hohe Wirkmächtigkeit zugesprochen werden muss - zum Beispiel hinsichtlich postpolitischer Formen des Regierens (Swyngedouw 2009) oder veränderter Arbeitswelten (Boltanski/Chiapello 2003), sondern auch, weil ihr eine eigene Logik des Kapitals zugrunde liegt. Jedoch ist diese Logik (und deren Folgen) wenn überhaupt, dann politisch gestaltbar und geht somit letztlich in der politischen Rationalität auf.

Durch die Berücksichtigung der emotionalen Rationalität wird der Stellenwert von Kapital oder von Arbeits- und Bodenmärkten bei Entscheidungen und Abwägungen in Planungsprozessen sowie bezüglich der Formen städtischen Regierens nicht geringer, aber mit ihr können die Handlungsbedingungen, unter denen sich Akteure an eine politische Bearbeitung dieser ökonomischen Rationalität machen, umfassender in 
den Blick genommen werden. Auch ziele ich nicht auf eine Logik, die in Endlichkeit und Begrenztheit der stofflich-natürlichen Umwelt gründet, da der Umgang mit ihr wiederum politischer Natur ist. Mit den vorgeschlagenen Ansätzen der ,dritten Generation' verlieren technische, kommunikative und politische Rationalitäten also nicht ihre Berechtigung. Die in diese Rationalitäten eingeschriebenen Utopien treten aber klarer hervor, indem die Logik der Emotionen an sie herangetragen wird. Gerade für gelungene kooperative Diskurse gilt es zum Beispiel deren emotionalen Vorbedingungen zu beachten. Hier ist unter anderem zu berücksichtigen, dass es einer in „nicht-politischen Kontexten eingeübte[n] Bereitschaft zur kooperativen Problembearbeitung“ bedarf (Weber 2012: 209). Letztlich macht der Einbezug von Emotionen die Analyse des Handelns von Planenden nicht einfacher, aber treffsicherer. Und hier liegt dann auch ein (notwendig) utopisches Moment der ,dritten Generation' selbst: die Annahme, Fühlen könnte ausreichend erforscht und beobachtet werden. Allein durch die reflexive Übersetzung des Fühlens in artikulierbare Gefühle wird die Einheitlichkeit des Erlebenszusammenhangs aufgelöst (Schützeichel 2012a: 231). Wenn Celikates (2009: 240) in Bezug auf „die Herstellung reflexiver Inakzeptabilität" von kritischer Theorie verlangt, dass sie „die Ebene der metatheoretischen Reflexion und der metakritischen Analyse der Bedingungen der Kritik verlassen und sich selbst ins „Handgemenge“ der kritischen Praxis begeben [muss]“, dann dürfte dies für das Erlangen eines Verständnisses der Gefühlslage(n) von in spezifischen Situationen Handelnden erst recht gelten - denn das konkrete Erfühlen bleibt einem theoretischen Zugang verwehrt. Für eine realistische Einschätzung der Schwierigkeiten politischen Handelns in der Planungspraxis müsste es also auch um das ungefilterte Anfühlen planungspraktischen Handelns gehen, bei dem trotz realpolitischer Widersprüche versucht wird, über diese hinauszureichen. Doch dies kann nur im konkreten Tun gelingen.

\section{Ausblick: Planungsansätze der, dritten Generation' - politisch gewendet}

Mit dem Gesagten ist ein Spannungsfeld aufgezeigt zwischen Planungspraxis, die im Vertrauen auf die Annehmbarkeit ihrer ,Produkte' gründet, und dem Moment der Irritation und Verunsicherung als Voraussetzung für Reflexivität und Rationalität. Welche Beiträge könnte Planung nun leisten, um „die Realität unannehmbar zu machen“ (Boltanski 2010: 21, Hervorh. i. Orig.) und so eine Funktion von kritischer Theorie ernst zu nehmen? Wie positioniert sie sich im Dilemma von kontinuierendem Handeln (um den Projektfortschritt zu sichern) und Handlungsunterbruch durch Irritation? Wie in jenem zwischen emotionaler Akzeptabilität als Innovationsbedingung und (reflexiv-)emotionaler Inakzeptabilität als Rationalitätsgebot? Denn wenn für einen erfolgreichen Transfer (sozialer) Innovationen diese als emotional annehmbar empfunden werden können bzw. müssen (Schröder et al. 2011: 29 ff.), dann liegt es in der jeweiligen Situation erst einmal nicht nahe, dass häretisch neue gegenüber eingespielten orthodoxen Ansätzen zum Tragen kommen. Ich vermute, dass bei 
der theoretischen Betrachtung von Planung im Verweis der Emotionen auf die hinteren Plätze genau dieses Dilemma zwischen nötigem Vertrauen und nötiger Verunsicherung zum Ausdruck kommt. Denn empirisch mangelt es an Quellenmaterial, insofern sich (lokal-)staatliche Planungspraxis notwendigerweise auf die eine Seite des Dilemmas zu schlagen scheint.

Doch müsste eine sich politisch verstehende Planung nicht gerade den gegenteiligen Weg einschlagen? Damit möchte ich nochmals auf den Kern von Planungsansätzen der ,dritten Generation“ hinweisen. Es geht nicht zumindest nicht primär - um eine Emotionalisierung von Planung, sondern um die Frage, wie in Planung mit Rationalitätsbedingungen politischen Handelns umgegangen wird. So wären mit den ersten beiden Schritten des Emotionslernens - Verunsicherung gefolgt von Information - auch emotionstheoretisch plausibilisierte Vorschläge für ein politisches Planen ausgewiesen. Diese Schritte könnten verstanden werden als Dekonstruktion heutiger gesellschaftlich hegemonialer Praxen und ihrer (Re-)Konstruktion als spezifisch solidarische Praxen. Nicht zuletzt könnte hier aus der Bewegungspraxis gelernt werden. So arbeitet beispielsweise die politische Aktionsform der ,Direct Action' konzeptionell mit dem Prinzip des Emotionslernens: Sie besteht „,idealtypisch aus dem Dreiklang von Aktion, entstehendem Erregungskorridor und inhaltlicher Füllung des Korridors“ (Direct-Action-Reader o. J.: 16).

Planungsansätze der, dritten Generation“ werden daher nicht nur auf die emotionale Dimension von Planung fokussieren, wo es beispielsweise um Anerkennungsstrukturen im Alltag geht, um Emotionseffekte von Institutionen, um Bildproduktionen, um mangelndes Vertrauen oder um Dissens bei Beteiligungsprozessen oder allgemein um Aushandlungsprozesse.[13] Mit entsprechenden Planungsansätzen könnten Planungsziele in den Blick genommen werden, die zum Beispiel auf ein Mehr an Empathiefähigkeit (Arnold 2005: 255) oder auf Subjekt- statt Instrumentalbeziehungen (Meretz 2011) setzen und nach gesellschaftlichen Praxen fragen, die geeignet sind, Kompetenzen solidarisch-kooperativen Handelns zu fördern. Mit solchen Planungsansätzen wird Planung - politisch verstanden - vor allem auch ihre eigenen Bedingungen zum Thema machen. Da bei projektbezogener Planungspraxis ein irritierendes Aus- und Unterbrechen aus dem Verwaltungsauftrag, der Regierung Lösungsvorschläge zu unterbreiten, (fast) nur oppositionell erwartet werden kann, müsste es politischer Planungspraxis um die Öffnung und Aneignung von Reflexionsräumen für (gesellschaftliche) Diskurse gehen. Dabei dürften Planende durchaus davon ausgehen, für urbane Raumproduktion nur bedingt verantwortlich zu sein dies vermag sie beim Aufwerfen grundlegender Fragen nicht zu Unrecht zu entlasten.[14] Was könnte dabei vorrangiges Thema solcher Diskurse sein?

Hier möchte ich an Oliver Marchart (2010: 362 f.) anknüpfen. Mit ihm muss in Bezug auf ein Solidaritätsprinzip eine sich als demokratisch bezeichnende Politik für die Ausschlüsse, die sie produziert, Verantwortung übernehmen. Solche Ausschlüsse gälte es zu thematisieren: Wer wurde beispielsweise mit demokratischen Entscheiden marginalisiert? Welche Folgen bleiben ausgeblendet? Damit müssten lokale als auch globale Wirkungen gesellschaftlichen Handelns berücksichtigt werden. So müssten zum Beispiel die in keiner Weise universalisierbaren gesellschaftlichen 
Naturverhältnisse des heutigen (de-)industrialisierten Nordens zum Thema gemacht werden. Dabei wäre der dystopische Charakter und die Verantwortungslosigkeit ,unserer ${ }^{\star}$ Lebensmuster erkennbar, erfahrbar und theoretisch verständlich zu machen. Was könnte solch potenziellen Momenten reflexiver und emotionaler Inakzeptabilität dann ergänzend als zweiter, informierender Schritt eines erfolgreichen Emotionslernens gegenübergestellt werden? Welche positiv besetzten Alternativen könnten in Diskussionen eingebracht werden?[15]

Hier liegt es nahe, dass in der Raumplanung auf gängige Leitbilder zurückgegriffen wird, wie zum Beispiel auf das der, nachhaltigen Entwicklung' oder jenes der ,Stadt der kurzen Wege'. Sollte es mit ihnen gelingen, die emotionale Inakzeptabilität wieder zu schließen, wäre für die Planungspraxis freilich viel gewonnen - nicht weniger, als dass im Wissen um diese Leitbilder eine unaushaltbare Welt wieder aushaltbar wird. Aus politischer Sicht wird damit auch aus einer emotionstheoretischen Warte zentral, ob es sich tatsächlich um glaubwürdige Leitbilder handelt. Sind ihre potenziell beruhigenden Wirkungen - die sie nicht nur gesellschaftlich, sondern auch auf Planende selbst entfalten können - normativ gut begründbar? Handelt es sich bei der Implementierung der Leitbildvorstellungen um effiziente Maßnahmen? Oder geht es nur um ein fortwährendes Spiel im Mix von Verunsicherung und Vertrauen in vorgeschlagene und im Laufe der Jahre modifizierte Lösungen, die aber jeweils nicht weitreichend genug sind? Zugespitzt: Weder ein Weiter-so und Nichtstun noch entschieden und radikal werden - das heißt emotional: weder Scham noch Angst? [16] Und sollte sich herausstellen, dass ein legitimatorischer Charakter von solchen Leitbildern überwiegt: Welche besseren Entwürfe urbanen Zusammenlebens könnten den durch die Irritation aufgemachten Möglichkeitsraum füllen?Letztlich ist Ziel des Emotionslernens, dass mit der dritten, transformativen Stufe bisherige Angstbewältigungsstrategien zugunsten eines weniger emotionalen „Sich-in-der-Welt-Fühlens“ aufgegeben werden und neue Erklärungsweisen geteilt werden können (Arnold 2005: 235, 224 ff.). Planungsansätze der ,dritten Generation“ könnten damit nicht zuletzt helfen, einen weniger gefühlsbetonten und stattdessen verstandesmäBigeren Zugang auf politisch zu klärende Fragen zu entwickeln.

\section{Endnoten}

[1] Im Jahr 2013 neu herausgegeben von Wolf D. Reuter und Wolfgang Jonas (vgl. Rittel 2013).

[2] Zu Beispielen des Machtgebrauchs vgl. Reuter 2000: 10 f., 1989: $151 \mathrm{ff}$.

[3] Dass es nachholende vertrauensbildende Maßnahmen schwer haben, verdeutlicht ,Stuttgart 21'; vgl. Schubert 2011a zur beruhigenden Funktion der Schlichtung zu ,Stuttgart 21'.

[4] Rittel (1977: 123 ff.) verweist als legitimierende, aber implizite Begründungen von Spontanurteilen auf etliche „Garantoren“ wie Tradition, Erfahrung, gute Sitten, Wissenschaft etc. In gewisser Weise sind diese mit den unterschiedlichen Rechtfertigungslogiken bei Boltanski/Thévenot (2007: 120 ff.) vergleichbar.

[5] Wie bei Schützeichel (2012a) verwende ich für diesen Beitrag Emotionen, Affekte und Gefühle als Arbeitsbegriffe synonym. Hammer-Tugendhat/ Lutter (2010: $10 \mathrm{ff}$.) dagegen betonen, dass je nach Fragestellung unterschiedliche Bedeutungsgehalte dieser 
Begriffe historisch und kulturell zu kontextualisieren sind. Roth (2009: 171) differenziert aus neurobiologischer Sicht zwischen Affekten und Emotionen.

[6] Auf entsprechende Diskrepanzerfahrungen als Handlungsmotivation verweisen zum Beispiel auch Rothermund (2011: 141, 151) oder Pettenkofer (2012: 210).

[7] Schützeichels Typologie erweist sich mit den Scheidungskriterien verschiedener Reflexivitäts- respektive Bewusstheitsgrade anschlussfähig auch an das Handlungsverständnis der „Pragmatik der Reflexion“ bei Boltanski/Thévenot (2007: 476) oder jenes der „Logik der Praxis“ in Verbindung mit einer „Ökonomie der Logik“ bei Bourdieu (1993: 157 f.) sowie an dessen Konzeption habituellen, großenteils unbewussten Handelns (ebd.: 114). Hierbei stellt hochreflexives Planungshandeln jeweils einen Grenzfall dar (vgl. Bourdieu/Wacquant 1996: 172).

[8] Busch verweist - aus einem kritischen Interesse der Akteure heraus - auf eine mögliche Funktion der Psychoanalyse, da durch diese „Einblicke ins Innenleben der Berufswelt“ möglich werden. Er sieht, dass eine „Repolitisierung“ der Subjekte für diese im politischen Feld eine „höchst belastende, anspruchsvolle Praxis“ mit sich bringt, bei der sie „auf Begleitung angewiesen“ sind (Busch 2012: 46 f.). Busch schlägt nichttherapeutische Formen der Gruppensupervision vor, um Gefühle, wie zum Beispiel Ängste, gemeinsam bearbeiten zu können.

[9] Vgl. z.B. die Beiträge in der Zeitschrift DISP (zumindest seit 2000, Jg. 36) und in Altrock et al. 2004 (so auch jene, die auf „die offenen Stellen“ [Fürst] respektive „Zukunftsperspektiven für die Planungstheorie“ [Altrock] zielen), sowie die Beiträge in Selle 2006a, 2006b und Konter 1998 (mit einem Überblick über Inhalts- und Problembereiche von Planungstheorie).

[10] Burckhardt (2004: 77) verweist mit Blick auf Planende zum Beispiel auf Aspekte wie Ruhm oder Wirkungen der „psychischen Situation des entscheidenden Subjekts innerhalb seines Amtes“.

[11] Vgl. z.B. Rittel 1988: 143, Renn 1996; Burckhardt (2004: 62) benennt unter anderem schichtspezifische Ziele, die von Planenden verfolgt werden.

[12] Ideale Diskursvoraussetzungen als Utopie der kommunikativen Rationalität dürfen nicht - wie dies häufig geschieht (vgl. z. B. Marchart 2010: 219) - auf konsensorientierte Deliberation verkürzt werden, sondern zielen mit Apel (1973: 432) auf die Aufhebung der Klassengesellschaft und mit Habermas (1994: 396 ff.) im Sinne einer „methodischen Fiktion “ auf ein Korrektiv im Rahmen der prozeduralen Weiterentwicklung von Gesellschaft als Rechtsgemeinschaft.

[13] Auch Deliberationsprozesse sind bezüglich ihres emotionalen Moments zu verstehen, da sie auf einem emotional vermittelten Symbolaspekt basieren, der letztlich bezweckt, „die Debatte über die zugrunde liegenden Werte vorläufig abzuschließen“ (Göhler 2012: 247), um zur Diskussion der eigentlichen Themen zu gelangen.

[14] Mit Habermas müsste der Vorwurf, Verwaltungen würden managen, statt politisch verantwortlich zu handeln, insofern als fehlgerichtet bezeichnet werden, als die Verantwortung, die Administrationen durch Gesetze mit unzähligen unbestimmten Rechtsbegriffen und hohen Ermessensspielräumen zugemutet wird, im Grunde von einem heute marginalisierten Gesetzgeber zu tragen wäre. Dieser kann freilich Kompetenzen delegieren, hätte dann aber zugleich „Legitimationsfilter“ für eine „Demokratisierung“ der Verwaltung“ einzubauen (Habermas 1994: 531, Hervorh. i. Orig.). Habermas verweist auf den Machtzuwachs der Verwaltungen in Deutschland und in den USA (ebd.: 233 ff., $516 \mathrm{ff}$.).

[15] Auf das Erfordernis positiver Konnotation verweisen zum Beispiel Honneth (2010: 120 ff.), Welzer und Rammler (2012: 14, 308) sowie Schröder et al. (2011: 34 ff.).

[16] Auch wenn ich den Nachweis hier nicht führen kann: Beide erwähnten Leitbilder können meines Erachtens mit Honneth als ungerechtfertigte „Ideologien der Anerkennung“ (2010: 103 ff.) ausgewiesen werden, da jeweils die ideelle und/oder materielle Glaubwürdigkeit nicht ausreichend gegeben ist (vgl. kritisch zu Nachhaltigkeit z.B. Spehr 1996, Groeneveld 1997, Swyngedouw 2009, Schubert 2012; zu Alternativen Adler/Schachtschneider 2010 und P.M. 2008). Zur Diskussion der ,Stadt der kurzen Wege‘ könnte in Analogie zu Žižek (2009: 92) gesagt werden, „weil sie stillschweigend die Sphäre der Geschwindigkeit von der Politisierung ausnimmt“ und damit eine erforderliche Modernekritik elegant unsichtbar gemacht wird (vgl. kritisch z.B. Pfleiderer/Braun 1995 und Schubert 2011b). 


\section{Autor_innen}

Axel Schubert, Dipl.-Ing. Arch., Bauassessor und Stadtplaner, arbeitet seit 2005 als Projektleiter im Planungsamt des Bau- und Verkehrsdepartements des Kantons Basel-Stadt, Abteilung Arealentwicklung und Nutzungsplanung.

schubert.axel@gmx.net

\section{Literatur}

Adler, Frank / Schachtschneider, Ulrich (2010): Green New Deal, Suffizienz oder Ökosozialismus? Konzepte für gesellschaftliche Wege aus der Ökokrise. München: Oekom Verlag.

Altrock, Uwe / Günther, Simon / Huning, Sandra / Peters, Deike (Hg.) (2004): Perspektiven der Planungstheorie. Berlin: Leue Verlag.

Apel, Karl-Otto (1973): Transformation der Philosophie, Bd. 2. Frankfurt a. M.: Suhrkamp Verlag.

Arnold, Rolf (2005): Die emotionale Konstruktion der Wirklichkeit. Beiträge zu einer emotionspädagogischen Erwachsenenbildung. Baltmannsweiler: Schneider Verlag Hohengehren.

Boltanski, Luc (2010): Soziologie und Sozialkritik - Frankfurter Adorno-Vorlesungen 2008. Berlin: Suhrkamp Verlag.

Boltanski, Luc / Chiapello, Ève (2003): Der neue Geist des Kapitalismus. Konstanz: UVK Universitätsverlag.

Boltanski, Luc / Thévenot, Laurent (2007): Über die Rechtfertigung. Eine Soziologie der kritischen Urteilskraft. Hamburg: Hamburger Edition.

Bourdieu, Pierre (1993): Sozialer Sinn. Kritik der theoretischen Vernunft. Frankfurt a. M.: Suhrkamp Verlag.

Bourdieu, Pierre (1998): Praktische Vernunft. Zur Theorie des Handelns. Frankfurt a. M.: Suhrkamp Verlag.

Bourdieu, Pierre / Wacquant, Loïc J.D. (1996): Reflexive Anthropologie. Frankfurt a. M. : Suhrkamp Verlag.

Burckhardt, Lucius (2004): Wer plant die Planung? Architektur, Politik und Mensch. Berlin: Martin Schmitz Verlag.

Busch, Hans-Joachim (2012): Psychoanalytische Politische Psychologie heute. Zwischenbilanz und Perspektiven. In: Markus Brunner / Jan Lohl / Marc Schwietring / Sebastian Winter (Hg.), Politische Psychologie heute? Themen, Theorien und Perspektiven der psychoanalytischen Sozialforschung. Gießen: Psychosozial Verlag, 33-50.

Celikates, Robin (2009): Kritik als soziale Praxis. Gesellschaftliche Selbstverständigung und kritische Theorie. Frankfurt a. M./New York: Campus Verlag.

Ciompi, Luc (2005): Mensch, Natur und Gefühl. Aus der Perspektive der fraktalen Affektlogik. In: Michael Gebauer / Ulrich Gebhard (Hg.), Naturerfahrung. Wege zu einer Hermeneutik der Natur. Kusterdingen: Die Graue Edition, 28-66.

Direct Action Reader (o. J.): Grundlagen und Tipps für konkrete Aktionen, o. O., unter: http://www.aktionsversand.de.vu/.

Fischbach, Rainer / Kahlen, Hans / Reuter, Wolf D. (1992): Notizen zur Bedeutung der Theorie- und Methodenlehre Horst Rittels für das Facility Management. In: Horst W.J. Rittel (1992), Planen Entwerfen Design: ausgewählte Schriften zu Theorie und Methodik, Stuttgart: Kohlhammer Verlag, 405-412.

Göhler, Gerhard (2012): Die affektive Dimension der Demokratie. Überlegungen zum Verhältnis von Deliberation und Symbolizität. In: Felix Heidenreich / Gary S. Schaal (Hg.), Politische Theorie und Emotionen. Baden-Baden: Nomos Verlag, 235-253.

Groeneveld, Sigmar (1997): Unterhalt statt Nachhaltigkeit. In: Werner G. Raza / Andreas Novy (Hg.), Nachhaltig reich - nachhaltig arm? Kritische Geographie 12. Wien: Brandes \& Apsel, 25-40.

Habermas, Jürgen (1994): Faktizität und Geltung. Beiträge zur Diskurstheorie des Rechts und des demokratischen Rechtsstaates. Frankfurt a. M.: Suhrkamp Verlag.

Hammer-Tugendhat, Daniela / Lutter, Christina (2010): Emotionen im Kontext. Eine Einleitung. In: Zeitschrift für Kulturwissenschaften (ZfK) 2/2010, 7-14. 
Heidenreich, Felix (2012): Versuch eines Überblicks: Politische Theorie und Emotionen. In: Felix Heidenreich / Gary S. Schaal (Hg.), Politische Theorie und Emotionen. BadenBaden: Nomos Verlag, 9-26.

Honneth, Axel (1992): Kampf um Anerkennung. Zur moralischen Grammatik sozialer Konflikte. Frankfurt a. M.: Suhrkamp Verlag.

Honneth, Axel (2010): Das Ich im Wir. Studien zur Anerkennungstheorie. Berlin: Suhrkamp Verlag.

Jessen, Johann / Reuter, Wolf D. (2006): Lernende Praxis. Erfahrung als Ressource planungstheoretische Konsequenzen. In: Klaus Selle (Hg.), Planung neu denken. Bd. 2: Praxis der Stadt- und Regionalentwicklung. Analysen, Erfahrungen, Folgerungen. Dortmund: Dorothea Rohn Verlag, 42-56.

Konter, Erich (1998): Planungstheorie und Planungsgeschichte. In: Erich Konter (Hg.), Planungspraxis und Planungstheorie: Festschrift für Karolus Heil. Berlin: Verlag für Wissenschaft und Forschung, 105-125.

Löw, Martina (2008): Soziologie der Städte. Frankfurt a. M.: Suhrkamp Verlag.

Marchart, Oliver (2010): Die politische Differenz. Berlin: Suhrkamp Verlag.

Marcuse, Peter (2013): Re-imagining the City critically. In: dérive, Zeitschrift für Stadtforschung 53, 9-15.

Meier-Seethaler, Carola (2001): Gefühl und Urteilskraft. Ein Plädoyer für die emotionale Vernunft. München: C.H. Beck Verlag.

Meretz, Stefan (2011): Was ist kritische Psychologie? In: Contraste: Monatszeitung für Selbstverwaltung 28 (319), 7 .

Nullmeier, Frank (2006): Politik und Emotion. In: Rainer Schützeichel (Hg.), Emotionen und Sozialtheorie. Disziplinäre Ansätze. Frankfurt a. M./New York: Campus Verlag, 84-103.

Pettenkofer, Andreas (2012): Von der Situation ergriffen. Emotionen in der pragmatistischen Tradition. In: Annette Schnabel / Rainer Schützeichel (Hg.), Emotionen, Sozialstruktur und Moderne. Wiesbaden: VS Verlag für Sozialwissenschaften, 201-226.

Pfleiderer, Rudolf / Braun, Lothar (1995): Kritik an der Bundesverkehrswegeplanung. In: Internationales Verkehrswesen 47/10, 609-614.

P.M. (2008): Neustart Schweiz. So geht es weiter. Zürich: Edition Zeitpunkt.

Renn, Ortwin (1996): Kooperativer Diskurs. Kommunikation in der Umweltpolitik. In: Klaus Selle (Hg.), Planung und Kommunikation. Wiesbaden/Berlin: Bauverlag, 101-112.

Reuter, Wolf D. (1989): Die Macht der Planer und Architekten. Stuttgart: Kohlhammer Verlag.

Reuter, Wolf D. (2000): Zur Komplementarität von Diskurs und Macht in der Planung. In: DISP: Dokumente und Informationen zur Schweizerischen Orts-, Regional- und Landesplanung 36 (141), 4-16.

Rittel, Horst W.J. (1972): Zur Planungskrise: Systemanalyse der „ersten und zweiten Generation“. In: ders. (1992), Planen, Entwerfen, Design: ausgewählte Schriften zu Theorie und Methodik. Stuttgart: Kohlhammer Verlag, 37-58.

Rittel, Horst W.J. (1976): Sachzwänge - Ausreden für Entscheidungsmüde? In: ders. (1992), Planen, Entwerfen, Design: ausgewählte Schriften zu Theorie und Methodik. Stuttgart: Kohlhammer Verlag, 271-281.

Rittel, Horst W.J. (1977): Urteilsbildung und Urteilsrechtfertigung. In: ders. (1992), Planen, Entwerfen, Design: ausgewählte Schriften zu Theorie und Methodik. Stuttgart: Kohlhammer Verlag, 113-133.

Rittel, Horst W.J. (1988): Die Denkweise von Planern und Entwerfern. In: ders. (1992), Planen, Entwerfen, Design: ausgewählte Schriften zu Theorie und Methodik. Stuttgart: Kohlhammer Verlag, 135-148.

Rittel, Horst W.J. (1992): Planen, Entwerfen, Design: ausgewählte Schriften zu Theorie und Methodik. Stuttgart: Kohlhammer Verlag.

Rittel, Horst W.J. (2013): Thinking design: transdisziplinäre Konzepte für Planer und Entwerfer, hrsg. von Wolf D. Reuter und Wolfgang Jonas. Basel/Berlin: Birkhäuser Verlag.

Rittel, Horst W.J. / Kunz, Werner (1972): Der Ansatz der Informationswissenschaften. In: Horst W.J. Rittel (1992), Planen, Entwerfen, Design: ausgewählte Schriften zu Theorie und Methodik. Stuttgart: Kohlhammer Verlag, 151-160. 
Rittel, Horst W.J. / Webber, Melvin (1973): Dilemmas in einer allgemeinen Theorie der Planung. In: Horst W.J. Rittel (1992), Planen, Entwerfen, Design: ausgewählte Schriften zu Theorie und Methodik. Stuttgart: Kohlhammer Verlag, 13-35.

Roth, Gerhard (2009): Aus Sicht des Gehirns. Frankfurt a. M.: Suhrkamp Verlag.

Rothermund, Klaus / Eder, Andreas (2011): Motivation und Emotion. Wiesbaden: VS Verlag für Sozialwissenschaften.

Schnabel, Annette (2012): Emotionen, Sozialstruktur und Moderne - ein spannungsvolles Wechselverhältnis. Zur Einleitung. In: Annette Schnabel / Rainer Schützeichel (Hg.), Emotionen, Sozialstruktur und Moderne, Wiesbaden: VS Verlag für Sozialwissenschaften, 9-27.

Schnabel, Annette / Schützeichel, Rainer (Hg.) (2012): Emotionen, Sozialstruktur und Moderne. Wiesbaden: VS Verlag für Sozialwissenschaften.

Schönwandt, Walter L. (1999): Grundriss einer Planungstheorie der „dritten Generation“. In: DISP: Dokumente und Informationen zur Schweizerischen Orts-, Regional- und Landesplanung 35 (137-137), 25-35.

Schönwandt, Walter L. (2002): Planung in der Krise? Theoretische Orientierungen für Architektur, Stadt- und Raumplanung. Stuttgart: Kohlhammer Verlag.

Schönwandt, Walter L. (2011): Probleme als Ausgangspunkt für die Auswahl und den Einsatz von Methoden. In: ARL (Hg.), Grundriss der Raumordnung und Raumentwicklung. Hannover: Verlag der ARL, 291-310 u. 368-377.

Schröder, Tobias / Huck, Jana / De Haan, Gerhard (2011): Transfer sozialer Innovationen. Eine zukunftsorientierte Fallstudie zur nachhaltigen Entwicklung. Wiesbaden: VS Verlag für Sozialwissenschaften.

Schubert, Axel (2011a): Lange Wege und starke Worte bei Stuttgart 21 - zugleich ein Plädoyer für K21-minus. Emanzipation als nötiges Gegenmittel gegen Geißlers nachhaltige Beruhigungspille. In: PNDonline 2/ 2011, unter: http://www.planung-neu-denken.de/content/ view/196/41.

Schubert, Axel (2011b): Postfossile Mobilität: die Wege sind langsam und steinig. In: Arch+ 43 (203), unter: http://www.archplus.net/download/artikel/3645/.

Schubert, Axel (2012): Von den Verengungsgefahren nachhaltigen Mainstreamings zum planungstheoretischen Erfordernis der Emanzipation. Nachhaltigkeitskritik und das Beispiel der Entwicklungsplanung Dreispitz. In: Matthias Drilling / Olaf Schnur (Hg.), Nachhaltige Quartiersentwicklung: Positionen, Praxisbeispiele und Perspektiven. Wiesbaden: VS Verlag für Sozialwissenschaften, 69-92.

Schützeichel, Rainer (2012a): Emotionen in Handlungen. Skizzen zu einer soziologischen Integration von Emotions- und Handlungstheorie. In: Annette Schnabel / Rainer Schützeichel (Hg.), Emotionen, Sozialstruktur und Moderne. Wiesbaden: VS Verlag für Sozialwissenschaften, 227-255.

Schützeichel, Rainer (2012b): „Structures of Feelings“ und Emotionsmilieus. Eine programmatische Forschungsskizze über den Zusammenhang von Emotionen und Sozialstruktur. In: Annette Schnabel / Rainer Schützeichel (Hg.), Emotionen, Sozialstruktur und Moderne. Wiesbaden: VS Verlag für Sozialwissenschaften, 473-484.

Selle, Klaus (Hg.) (2006a): Planung neu denken. Bd. 1: Zur räumlichen Entwicklung beitragen: Konzepte. Theorien. Impulse, Dortmund: Dorothea Rohn Verlag.

Selle, Klaus (Hg.) (2006b): Planung neu denken. Bd. 2: Praxis der Stadt- und Regionalentwicklung: Analysen, Erfahrungen, Folgerungen. Dortmund: Dorothea Rohn Verlag.

Siebel, Walter (2006): Wandel, Rationalität und Dilemmata der Planung. In: Klaus Selle (Hg.), Planung neu denken. Bd. 1: Zur räumlichen Entwicklung beitragen: Konzepte. Theorien. Impulse. Dortmund: Dorothea Rohn Verlag, 195-209.

Slaby, Jan (2011): Affektive Intentionalität - Hintergrundgefühle, Möglichkeitsräume, Handlungsorientierung. In: Jan Slaby / Achim Stephan / Henrik Walter (Hg.), Affektive Intentionalität. Beiträge zur welterschließenden Funktion der menschlichen Gefühle. Paderborn: Mentis Verlag, 23-48.

Spehr, Christoph (1996): Die Ökofalle. Nachhaltigkeit und Krise. Wien: Promedia Verlag.

Stickler, Armin (2011): Organisation und soziale Bewegung. Zur Kritik der bewegungswissenschaftlichen Institutionalisierungsdebatte. In: Michael Bruch / Wolfram Schaffar / Peter Scheiffele (Hg.), Organisation und Kritik. Münster: Westfälisches Dampfboot, $105-136$.

Swyngedouw, Erik (2009): Immer Ärger mit der Natur: „Ökologie als neues Opium fürs Volk“. In: PROKLA. Zeitschrift für kritische Sozialwissenschaft 39/3 (156), 371-389. 
Weber, Florian (2012): Unterkühlter Diskurs. Zum Verhältnis von Emotion und Deliberation bei Jürgen Habermas. In: Felix Heidenreich / Gary S. Schaal (Hg.), Politische Theorie und Emotionen. Baden-Baden: Nomos Verlag, 199-215.

Welzer, Harald / Rammler, Stephan (Hg.) (2012): Futur Zwei. Die Wiedergewinnung der Zukunft. Frankfurt a. M.: Fischer Verlag.

Žižek, Slavoj (2009): Ein Plädoyer für die Intoleranz. Wien: Passagen Verlag.

\section{Emotional Rationality and Planning - Planning Approaches of a '3rd Generation'. Or: About the depoliticizing Potential of Trust-Building and Self-Assurance}

The article is based on the assumption that dealing with uncertainties is crucial to planning, because they threaten to interrupt the planning progress. Therefore, trust in assumptions and planning-products (plans, overall concepts etc.) from all those being involved is very important for planning practice. Emotional theory, by contrast, considers uncertainties and irritations as prerequisites for rationality, reflexivity and learning processes. That is why trust-building effects of planning have to be seen critically, when they tend to hide reflections and therefore also 'the Political'. With my article I focus on an understanding how planning practice deals with uncertainty. For this purpose I question given concepts and highlight the role of emotions in (planning) practice. Referring to an 'emotional rationality', I propose planning approaches of a 'third generation' and focus on the meaning, they could have for critical planning practice. I also want to contribute to an 'emotional turn' in planning theory. 